\title{
Novel fully-integrated biosensor for endotoxin detection via polymyxin B immobilization onto gold electrodes
}

\author{
A. Zuzuarregui ${ }^{1}$, S. Arana ${ }^{1,2}$, E. Pérez-Lorenzo ${ }^{1,2}$, S. Sánchez-Gómez ${ }^{3, *}$, G. Martínez de Tejada ${ }^{3}$, and \\ M. Mujika ${ }^{1,2}$ \\ ${ }^{1}$ CEIT and Tecnun (University of Navarra), Paseo Manuel Lardizábal No. 15, 20018 San Sebastián, Spain \\ ${ }^{2}$ CIC microGUNE, Goiru Kalea 9, 20500 Arrasate-Mondragon, Spain \\ ${ }^{3}$ Department of Microbiology and Parasitology, University of Navarra, Irunlarrea 1, 31008, Spain \\ *current address: Immunoadjuvant unit, Bionanoplus, Noain, Spain
}

Correspondence to: A. Zuzuarregui (anazuzuarregui@gmail.com)

Received: 16 May 2013 - Revised: 6 September 2013 - Accepted: 6 September 2013 - Published: 24 September 2013

\begin{abstract}
In this paper an electrochemical endotoxin biosensor consisting of an immobilized lipopolysaccharide (LPS) ligand, polymyxin B (PmB), is presented. Several parameters involved both in the device fabrication and in the detection process were analyzed to optimize the ligand immobilization and the interaction between PmB and LPS, aiming at increasing the sensitivity of the sensor. Different electrochemical pre-treatment procedures as well as the functionalization methods were studied and evaluated. The use of a SAM (self-assembled monolayer) to immobilize $\mathrm{PmB}$ and the quantification of the interactions via cyclic voltammetry allowed the development of a robust and simple device for in situ detection of LPS. Thus, the biosensor proposed in this work intends an approach to the demanding needs of the market for an integrated, portable and simple instrument for endotoxin detection.
\end{abstract}

\section{Introduction}

Sepsis is one of the most dreaded medical conditions, since it claims 750000 lives per year in the United States (Wang et al., 2010). This pathology may lead to extensive injury of vascular endothelium, which often results in a more severe syndrome known as septic shock (Annane et al., 2005). About $50-60 \%$ of septic shock episodes are related to infections by Gram-negative bacteria, and more concretely to the lipopolysaccharide (LPS or endotoxin), a major component of the bacterial outer membrane (Chaby, 1999). The human body is extremely sensitive to endotoxin and minimum amounts of this substance injected into it can cause serious effects to the system (e.g., fever, intravascular blood clotting and multiorgan failure) (Cohen, 2002).

Endotoxins are ubiquitous in the environment and they may be present in medical implants as well as in drugs for parenteral administration; so it is necessary to ensure that the content of endotoxin in these items does not exceed certain limits. As set by the American and European Pharmacopoeias, these limits range from 0.2 to 5 endo- toxin units (EU)/kg body/hour for intravenous injections and 2.15-20EU for medical devices (European Pharmacopeia, 2005; USPC, 2005), being $1 \mathrm{EU}$ approximately equivalent to $100 \mathrm{pg}$ of $E$. coli LPS. Therefore, the implementation and validation of efficient endotoxin detection techniques is of extreme importance.

There are two main validated methods for endotoxin detection nowadays: the test Limulus amebocyte lysate (LAL) and the in vitro pyrogen test (IPT). The LAL test, the most recommended by pharmacopoeias, is based on the coagulation cascade that the LPS triggers upon interaction with the horseshoe crab (Limulus polyphemus) amebocytes. The IPT measures the interleukin- $1 \beta$ secreted by human blood cells in the presence of LPS (Daneshian et al., 2008). Above this, there are several commercialized kits for endotoxin detection such as Pyrogent ${ }^{\mathrm{TM}}$, Endosafe ${ }^{\circledR}$ and EndoLISA ${ }^{\circledR}$. The former two systems are based on LAL reagents, whereas in the third kit the amount of endotoxin is quantified monitoring the recombinant Factor $\mathrm{C}(\mathrm{rFC})$. All these methods, despite being sensitive, require long incubation times to render 


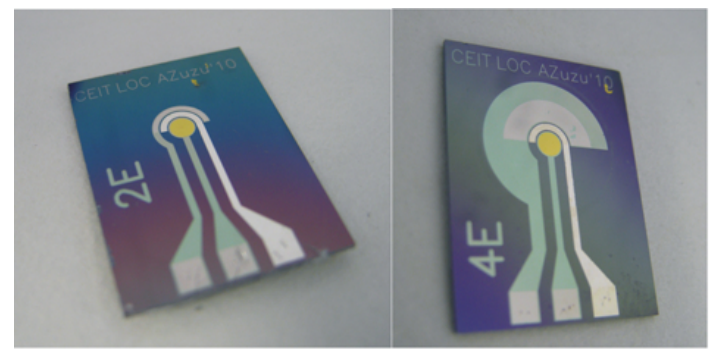

$$
G A R=\frac{\text { Area }_{C E}}{\text { Area }_{W E}}
$$

\begin{tabular}{|c|c|}
\hline Sensor & GAR \\
\hline 1 & 1.85 \\
2 & 14.15 \\
\hline
\end{tabular}

(A)

Figure 1. (A) Fabricated chips: sensor 1 and sensor 2, (B) geometry area ratio, (C) GAR values.

results and are costly since they use colorimetric or fluorometric techniques.

Groups all over the world are focusing their research on alternative sensing methods for endotoxin detection to outweigh those disadvantages (Hreniak et al., 2004; Limbut et al., 2007; Priano et al., 2007). The electrochemical approach based on sensors or biosensors stands out among all of them. Most of these studies rely on electrochemical impedance spectroscopy (EIS) for the LPS detection (Cho et al., 2012; Ding et al., 2007; Heras et al., 2010; Rahman et al., 2013). For instance, the sensors developed by Ding and his co-workers and Rahman and its group use EIS to quantify the amount of LPS through immobilized PmB (polymyxin B). Other electrochemical techniques, such as differential pulse voltammetry (DPV) or cyclic voltammetry (CV), have also been used for the implementation of endotoxin detectors (Kato et al., 2007; Yeo et al., 2011).

As far as we know, all these methods use macroelectrodes or setups, making the portability almost impossible. The alternative we propose is based on the application of microbiosensors to endotoxin detection. These devices allow realtime analysis of biological reactions and can be applied to nearly any field. This kind of sensors, fabricated by means of microsystem techniques, are analytical devices that consist of two main components: a bioreceptor and a transducer that turns the detection event into an electrical output which is instantly measured (Berganza et al., 2007). The integration of the three electrodes and the use of thin-film technologies provide the developed system with the miniaturization and portability needed to carry out field experiments while decreases both the fabrication and operation costs.

It is known that some polycationic molecules such as the antibiotic PmB bind with high affinity to LPS, which is negatively charged at neutral $\mathrm{pH}$ (Brandenburg, 2002). In addition to being cationic, $\mathrm{PmB}$ has an amphiphilic nature due to the simultaneous presence in the molecule of the polycationic ring and the hydrophobic chain. This enables the stoichiometric PmB-LPS interaction and makes their binding reversible, under appropriate conditions (Morrison and Jacobs, 1976). All these features make PmB a promising candidate ligand for LPS in a bioreceptor aimed at detect- ing endotoxin (Chang, 1997). To ensure the correct immobilization of the ligand and to avoid nonspecific adsorption of endotoxin onto the surface of the sensors, PmB was covalently linked to self-assembled monolayers (SAMs) previously formed on the gold electrodes (Ignat et al., 2010). SAMs are organized structures of organic molecules that allow the efficient and simple immobilization of different compounds used for biological detection (Pillay et al., 2009). Once SAMs are formed, they remain strongly attached to the surface through their terminal thiol group and provide a well-defined and stable interface for ligand immobilization (Ansorena et al., 2011).

The aim of this study is the fabrication and characterization of an alternative method for endotoxin quantification. The microsensor proposed here is based on the detection of LPS via CV using PmB immobilized through a SAM as ligand. The integration of the whole device in a compact cell and the use of electrochemical techniques provide the basis for the development of a novel and in situ endotoxin detection method that the market is demanding.

\section{Material and fabrication methods}

\subsection{Sensor fabrication}

As an electrochemical biosensor, the device developed in this work consists of three integrated microelectrodes: a working electrode (WE), a counter electrode (CE) and a pseudoreference electrode (RE). To analyze the influence of the geometry of the $\mathrm{CE}$ in the performance of the WE, two sensors differing in CE geometry were fabricated and tested (Fig. 1a). To quantify the influence of the area in the measurements, the geometry area ratio between CE and WE (GAR) was calculated according to Fig. 1b. The GAR values for the two sensors are shown in Fig. 1c.

Biosensors were fabricated employing standard microsystem processes on 4 in oxidized silicon wafers. The CE was made of platinum by DC sputtering (Edwards ESM-100) and patterned as shown in Fig. 1a with a thickness of $200 \mathrm{~nm}$. The WE, made of gold, was deposited by RF sputtering (Edwards ESM-100) and shaped as a disk of $1.8 \mathrm{~mm}$ of diameter 


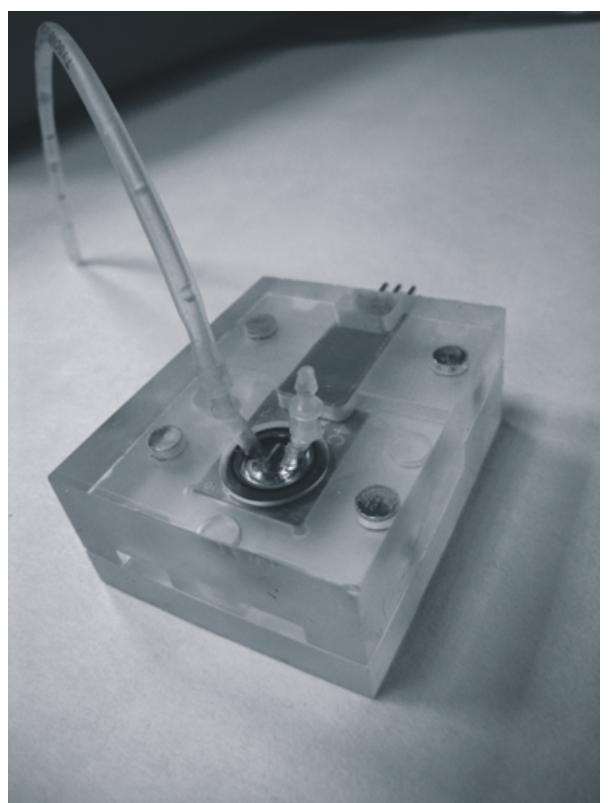

Figure 2. Biosensor mounted into the measurement cell. The inlet and outlet tubes can be appreciated

with a thickness of $150 \mathrm{~nm}$. With the aim of integrating the reference electrode inside the structure of the microdevice, a concentric semi-circle of silver was deposited by DC sputtering (Edwards ESM-100). This layer of $1000 \mathrm{~nm}$ of thickness works as a pseudo-reference electrode (Añorga and Arana, 2011). To protect all the inactive parts of the biosensors, a $600 \mathrm{~nm}$ coating of $\mathrm{SiO}_{2}$ was deposited by plasma enhanced vapor deposition (Oxford Plasmalab 80 plus). In order to fit the required sizes of the passivation layer, sensors were subjected to a wet $\mathrm{SiO}_{2}$ etching.

\subsection{Reaction cell fabrication}

To minimize the influence of environmental conditions in the experiments and to assure the cleaning and sterilization needed, a measurement cell was designed and fabricated (Fig. 2). This device is made of methacrylate and has a $40 \mu \mathrm{L}$ chamber where the assays take place. The base and cover of the cell are kept together with magnets and the microchamber is sealed by a toric joint. This microchamber can be filled and emptied using two fluidic connectors attached to the top of the cell.

\subsection{Reagents}

Sulfuric acid $\left(\mathrm{H}_{2} \mathrm{SO}_{4}\right)$, pyrogen free water, polymyxin B sulfate salt $(\mathrm{PmB})$ and phosphate buffer solutions (PBS) were purchased from Sigma-Aldrich. Perchloric acid $\left(\mathrm{HClO}_{4}\right)$, acetone $99.5 \%$ pure and ethanol $99.5 \%$ pure were supplied by Panreac. Mercaptopropionic acid (MPA), 1-ethyl3-(3-dimethylaminopropyl) carbodiimide (EDC) and N- hydroxysuccinimide (NHS) were purchased from Thermo Scientific. Trichlorethylene was obtained from Alden. Hellmanex II was supplied by Hellma. Ultra pure water of resistivity 18.2 M $\Omega$ was obtained from a Milli-Q Water System (Millipore Corp.).

\subsection{Lipopolysaccharide preparation}

LPS of E. coli ATCC 35218 was obtained from the aqueous phase of a water-phenol extract according to a published procedure (Leong et al., 1970). To remove traces of nucleic acids or proteins that could interfere with endotoxin detection, LPS extracts were dialyzed, lyophilized and purified following published protocols (Hirschfeld et al., 2000). The LPS from $E$. coli is the endotoxin used as reference by the regulatory agencies.

\section{Experimental process}

The whole electrochemical experiments were monitored with the Autolab Potentiostat PGSTAT 128N using the Nova 1.6 software version (Eco Chemie).

\subsection{Sample preparation}

Prior to conducting any procedure and to avoid the interference from potential contaminants, the sensors were subjected to a standard cleaning process consisting of three $5 \mathrm{~min}$ sonication steps, first in trichlorethylene, then in acetone and finally in ethanol.

To clean and prepare the sensors for the ligand immobilization, the gold electrodes ( $1.8 \mathrm{~mm}$ diameter) were electrochemically activated via cyclic voltammetry (CV). For this purpose, the potential was scanned from 0 to $1.2 \mathrm{~V}$ with a $0.1 \mathrm{~V} \mathrm{~s}^{-1}$ scan rate. Two electropolishing solutions were tested: sulfuric acid $0.05 \mathrm{M}$ and perchloric acid $1 \mathrm{M}$. After each voltammetry assay, electrodes were rinsed with deionized water and dried with $\mathrm{N}_{2}$.

SAMs were formed on the gold surface via a thiol group. The electrodes were incubated in MPA $1 \mathrm{mM}$ for $2 \mathrm{~h}$ and then rinsed with ethanol to remove nonspecific bindings. To ensure ligand immobilization it is necessary to activate the SAM. For this purpose, the electrodes were incubated first with EDC $46 \mathrm{mM}$ for $1 \mathrm{~h}$ and then with NHS $46 \mathrm{mM}$ for $1 \mathrm{~h}$. After each step, the microchamber was washed with water to remove unbound molecules.

Once the SAM was activated, the ligand was immobilized onto the working electrode surface. For this aim, the chamber was incubated with $100 \mu \mathrm{L}$ of a $100 \mu \mathrm{gmL}$ solution of PmB for $2 \mathrm{~h}$. Before the subsequent processes, the chamber was rinsed to minimize nonspecific binding of the ligand.

LPS molecules form biologically active aggregates and the rate of aggregation can differ between experiments. To reduce this source of variability endotoxin containing solutions were subjected to three consecutive cycles of heating ( $10 \mathrm{~min}$ 
at $56^{\circ} \mathrm{C}$ ) and cooling $\left(2 \mathrm{~min}\right.$ at $\left.-20^{\circ} \mathrm{C}\right)$ employing a vortex mixing between steps. To quantify the LPS detection by the functionalized sensor, $100 \mu \mathrm{L}$ of the solution were placed in the measurement chamber for $30 \mathrm{~min}$. Three different concentrations of analyte were tested: 100,10 and $1 \mu \mathrm{g} \mathrm{mL}^{-1}$.

As control assays, experiments without SAM were carried out to analyze the need for ligand immobilization. In these assays, PmB was immobilized directly onto the gold surface and then the LPS was added.

After performing the assays, the sensors, the cell chamber and the connectors were washed with Hellmanex II and then incubated with the same compound for $30 \mathrm{~min}$. Finally, both sensor and cell were rinsed thoroughly with deionized water and dried with $\mathrm{N}_{2}$.

\subsection{Cyclic voltammetry measurements}

The influence of the electrode's geometry, the gold pretreatment and the validation of the detection system were carried out by means of cyclic voltammetry (CV). All the assays were performed in the same environmental conditions: at room temperature $\left(23^{\circ} \mathrm{C}\right)$ and in PBS buffer $(\mathrm{pH}$ 7.4). The $\mathrm{CV}$ measurements were carried out in the range -0.2 to $0.7 \mathrm{~V}$, with a scanning rate of $0.1 \mathrm{~V} \mathrm{~s}^{-1}$.

Each assay consisted of three measurements. First of all, the behavior of the working electrode prior to any immobilization step was characterized (Bare Gold). Then another $\mathrm{CV}$ reading after the $\mathrm{PmB}$ immobilization was performed to monitor any potential change. Finally, the response of the sensor after the LPS incubation was measured, to analyze the interaction of the two molecules and quantify the amount of endotoxin bound.

\section{Results and discussion}

As previously mentioned, the purpose of this work was to design, fabricate and test an endotoxin biosensor. The results obtained are summarized in three sections focused on the following issues: the analysis of the influence of the CE geometry, the study of different solutions for gold pre-treatment and, finally, the validation of the performance of the biosensor.

\subsection{Effect of the geometry of the counter electrode}

The fabrication and patterning of the CE has a significant influence on the WE performance and on the degradation of the $\mathrm{CE}$ itself (due to the current densities passing through the platinum layer) as some authors have shown (Kim et al., 2004; Radev et al., 2010). This fact led us to design and fabricate two types of sensors varying the CE area. To compare the performance of the two developed sensors (Fig. 1a), experiments involving the formation of the SAM followed by the addition of PmB $\left(100 \mu \mathrm{g} \mathrm{mL}^{-1}\right)$ and LPS $\left(100 \mu \mathrm{g} \mathrm{mL}^{-1}\right)$

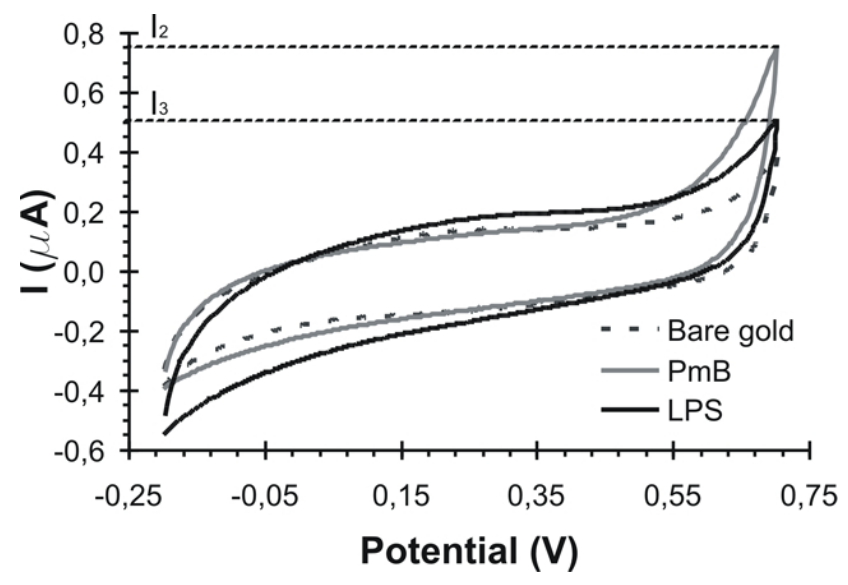

Figure 3. Cyclic voltammogram $(-0.2-0.7 \mathrm{~V})$ of the bare gold, the immobilized $\mathrm{PmB}\left(100 \mu \mathrm{gmL}^{-1}\right)$ and the detected LPS $\left(100 \mu \mathrm{g} \mathrm{mL}^{-1}\right)$.

were carried out with the two types of biosensors. Figure 3 shows the results obtained in each assay.

As depicted in Fig. 3, the immobilization of PmB induced an increase in the signal measured, more than likely due to the conductive properties of this peptide. However, after the addition of LPS, the current dropped, reflecting both neutralization of the positive charge of the PmB by the LPS and the presence of electrically insulating groups (lipid A) in the lipopolysaccharides.

Considering that no redox couple is added, and therefore there is no possibility for electron transfer between the solution and the electrode, the amount of endotoxin detected is quantified in terms of the changes in the measured current after the immobilization of the PmB (measurement $I_{2}$ ) and once the LPS is detected (measurement $I_{3}$ ). The current changes are calculated according to Eq. (1) and shown in Fig. 4.

$\Delta I=\frac{I_{2}-I_{3}}{\text { Area }_{\mathrm{WE}}}$

Change in the measured current $(\Delta I)$ where Area ${ }_{W E}$ is the active area of the working electrode. These results imply that sensor 2 has a more sensitive response to LPS than sensor 1, since the change in current is significantly higher in the former. This conclusion is consistent with the fact that sensor 2 has a higher GAR value and that an increase of the GAR value improves the efficiency of the WE and prevents the deterioration of the CE. Therefore, sensor 2 was selected for subsequent assays.

\subsection{Influence of the electropolishing solution}

To investigate the effect of the medium used for the pretreatment of the working electrode, cyclic voltammetry experiments $(0-1.2 \mathrm{~V})$ were performed using two solutions: 


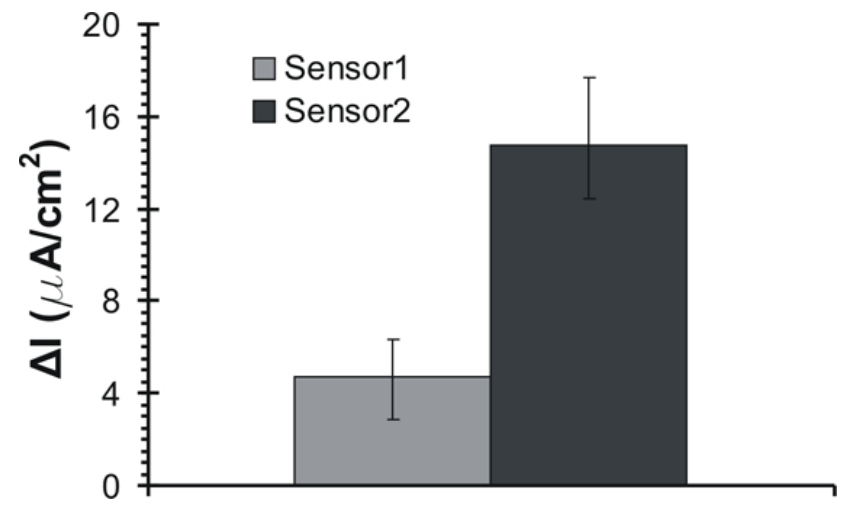

Figure 4. Analysis of the influence of the CE geometry. Change of the measured current in the two types of sensors due to the LPS $\left(100 \mu \mathrm{g} \mathrm{mL}^{-1}\right)$ interaction with the $\mathrm{PmB}\left(100 \mu \mathrm{g} \mathrm{mL}^{-1}\right)$ immobilized via SAM.

sulfuric acid $(0.05 \mathrm{M})$ and perchloric acid $(0.05 \mathrm{M})$. To compare both methods, once the pre-treatment was finished, all sensors were cycled once more in sulfuric acid solution, obtaining the usual voltammograms (Fig. 5). The assays for each solution were carried out with at least five different sensors to confirm the results.

To study the surface roughness of the gold layers the electrochemical surface area (ESA) of the electrodes after the different pre-treatment methods has been determined. The measurement of the oxygen adsorption has been chosen as indicator of the microscopic surface area of gold (Hoogvliet et al., 2000). This determination was done integrating the gold oxide reduction peak from the voltammetry curves referred to the electrode area $\left(Q_{\exp }\right)$. The standard reference charge of gold electrodes is $390 \pm 10 \mu \mathrm{Ccm}^{-2}\left(Q_{\text {std }}\right)$ (Trasatti and Petrii, 1991). The ESA is the ratio between the experimental charge of the gold electrodes and the theoretical one; whereas the roughness factor is that value expressed per unit of geometric surface area (Carvalhal et al., 2005).

$\mathrm{ESA}=Q_{\text {exp }} / Q_{\text {std }}$

The electrochemical surface area determined for the electrodes pre-treated with sulfuric acid is $3.52 \times 10^{-2} \pm 1.6 \times$ $10^{-3} \mathrm{~cm}^{2}$ (mean value and standard deviation of five electrodes) and the roughness factor $\left(R_{\mathrm{f}}\right)$ takes the value of $1.75 \pm 0.08$. On the other hand, the electrodes pre-treated with perchloric acid had an ESA of $2.83 \times 10^{-2} \pm 0.9 \times 10^{-3} \mathrm{~cm}^{2}$ (mean value and standard deviation of five electrodes) and a roughness factor $\left(R_{\mathrm{f}}\right)$ of $1.41 \pm 0.05$. These results improve the values obtained by other research groups (Bonroy et al., 2004; Carvalhal et al., 2005) with high reproducibility and confirm the increase in the active area that provides the gold electrodes with better biosensing features.

Despite these observations, we decided to study whether the pre-treatment of the sensor with either $\mathrm{H}_{2} \mathrm{SO}_{4}$ or $\mathrm{HClO}_{4}$ could influence its subsequent ability to interact first with

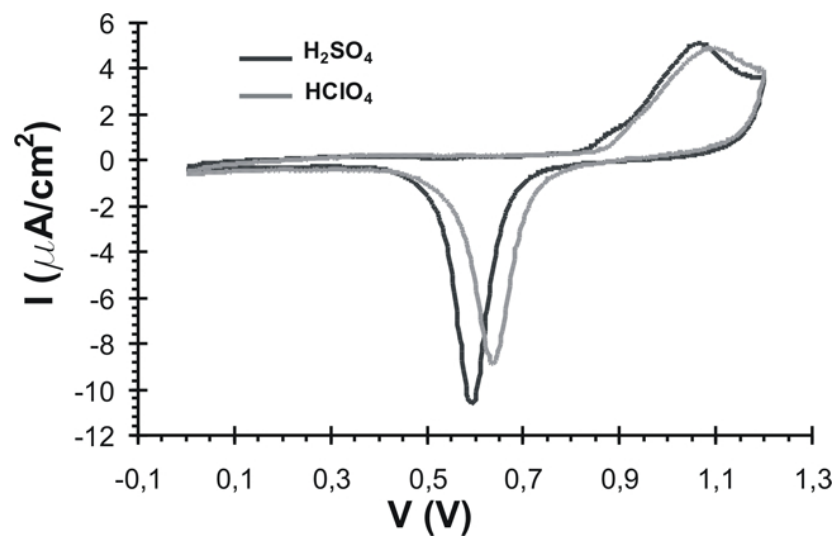

Figure 5. Cyclic voltammogram of the gold electrodes after $\mathrm{H}_{2} \mathrm{SO}_{4}$ and $\mathrm{HClO}_{4}$ electropolishing.

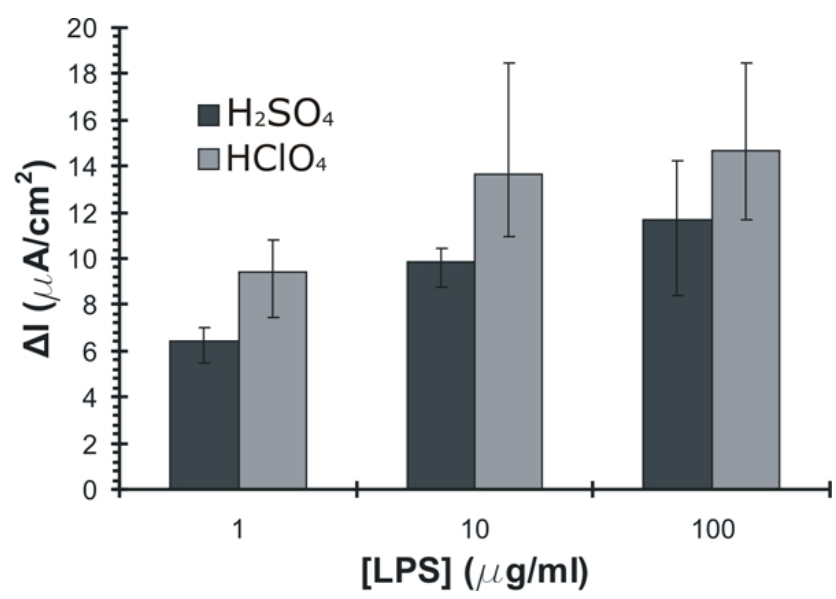

Figure 6. Influence of the pre-treatment solutions in the LPS detection. Change of the measured current in the two types of electropolished sensors due to the LPS interaction with the PmB $\left(100 \mu \mathrm{g} \mathrm{mL}^{-1}\right)$ immobilized via SAM.

$\mathrm{PmB}$ and then with LPS. For this purpose, sensors were first cycled with either $\mathrm{H}_{2} \mathrm{SO}_{4}$ or $\mathrm{HClO}_{4}$, and once the SAM formation and the $\mathrm{PmB}$ immobilization were performed, the amount of LPS bound to the sensor was quantitatively assessed. In these assays, PmB was added to the chamber at a concentration of $100 \mu \mathrm{g} \mathrm{mL}^{-1}$ and then different concentrations of LPS $\left(100 \mu \mathrm{g} \mathrm{mL}^{-1}, 10 \mu \mathrm{g} \mathrm{mL}^{-1}\right.$ and $\left.1 \mu \mathrm{g} \mathrm{mL}^{-1}\right)$ were tested. The results of this group of assays are shown in Fig. 6.

The data depicted in Fig. 6 confirm previous results with the voltammograms. Despite the fact that the absolute values obtained with the $\mathrm{HClO}_{4}$ pre-treatment are higher, this method results in an increase of the experimental variability. This makes almost impossible to discriminate between the sensor responses to $10 \mu \mathrm{g} \mathrm{mL}^{-1}$ of LPS and to $100 \mu \mathrm{g} \mathrm{mL}^{-1}$. In contrast, the use of $\mathrm{H}_{2} \mathrm{SO}_{4}$ results in lower variability and in clear dose-response behavior that allows differentiating the three concentrations of LPS tested. Ding and his co-workers stated that the pre-treatment with perchloric acid leads to 


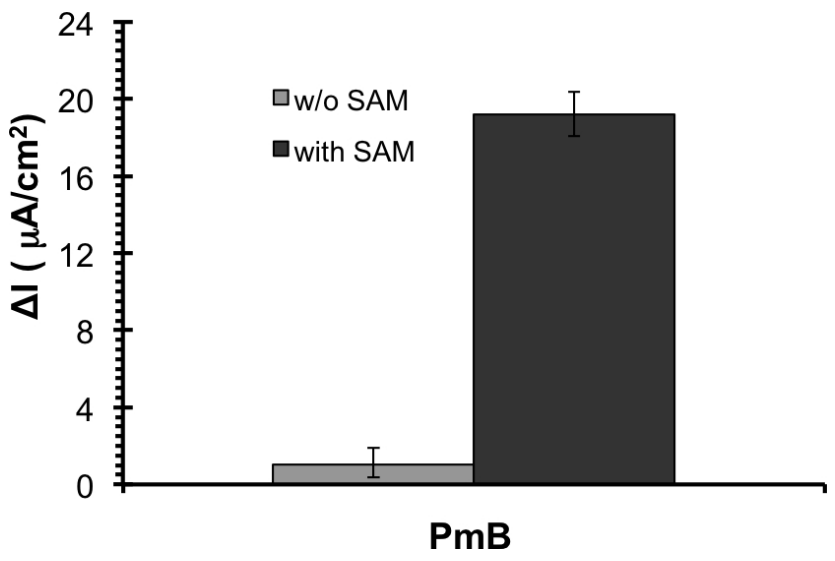

Figure 7. Effect of the SAM in the ligand immobilization. Change of the measured current due to the PmB immobilization with and without SAM.

better results than the sulfuric acid in their paper (Ding et al., 2007). They analyze the performance of the sensor through $\mathrm{CV}$ in $\mathrm{Fe}(\mathrm{CN})_{6}^{-3 /-4}$ measuring the obtained current, showing the voltammogram of a single sensor. Those results are similar to the ones obtained in this work as they get a larger response of the sensor; however, an increase in the current does not imply a better efficiency as shown in Fig. 6. These results together with the ESA and $R_{\mathrm{f}}$ values calculated from the cyclic voltammograms led us to select the sulfuric acid pre-treatment for subsequent assays.

\subsection{Test and validation of the device}

\subsubsection{Effect of the SAM in the PmB immobilization and LPS detection processes}

In order to test if the formation of SAM was necessary for an efficient PmB immobilization, the amount of PmB bound to sensors with and without SAM was quantified. In these assays, $\mathrm{PmB}$ was added at $100 \mu \mathrm{g} \mathrm{mL}^{-1}$ (Fig. 7).

Sensors with SAM bound approximately 10 times more $\mathrm{PmB}$ than their counterparts without SAM and the current increase due to $\mathrm{PmB}$ in sensors without SAM was almost imperceptible, demonstrating the importance of SAM formation for the proper PmB immobilization and presumably for an appropriate LPS detection. Without SAM, the PmB does not attach well to the gold surface and it might get washed off during the measurement process.

This behavior can be extrapolated to the LPS molecules as they do not include any sulfide group and have the mentioned amphiphilic character similar to the PmB. So, it can be presumed that the endotoxins do not get attached to the gold surface that could be available and do not interfere in the analysis. Regarding the possible binding to the SAM, LPS molecules do not have available amine groups so, as the immobilization takes places through a peptide bond, the reaction between the lipopolysaccharide and the self-assembled

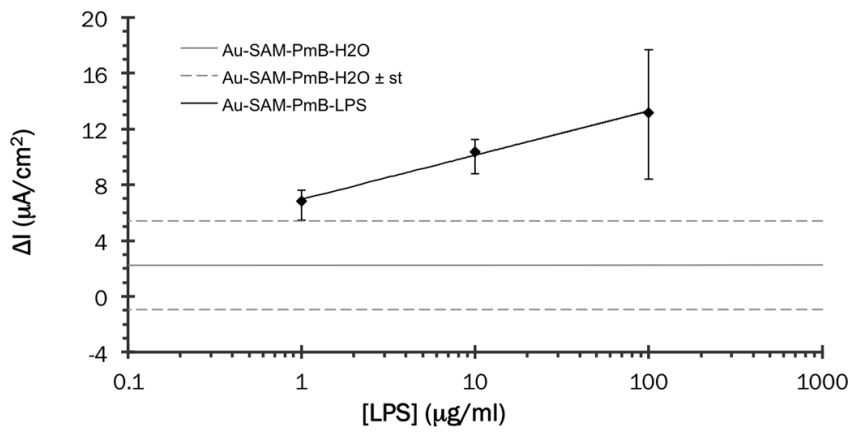

Figure 8. Quantitative detection of LPS by the biosensor.

monolayer is unlikely to happen. Besides this, the negative charge of the endotoxins and the SAM results in a repelling force that prevents the LPS binding.

\subsubsection{Quantitative analysis of LPS detection}

To demonstrate if the device allows detecting LPS quantitatively, the response of the optimized sensor (with SAM and $\mathrm{PmB}$ ) to decreasing concentrations of LPS was measured. Besides this, control assays with pyrogen-free water were made to establish the detection limit (grey continuous and dashed lines). The results of these assays are presented in Fig. 8.

Since the variability of the measurements decreases as the concentration of the analyte diminishes (from 35 to $12 \%$ ), these results show that the developed biosensor is suitable for LPS detection, particularly at low LPS concentrations. Interestingly, as shown in Fig. 8 the change in current is in a linear relationship with the LPS concentration in the range of concentrations tested. The LOD (limit of detection), calculated as the concentration that gives a signal three times the standard deviation of the background signal of the system, is $32.89 \mu \mathrm{g} \mathrm{mL}^{-1}$. Although lower detection limits have been reported for endotoxin detection; to the best of our knowledge, all the methods require macroelectrodes that prevent the miniaturization of the device and hamper their portability. Therefore, the microbiosensor developed in this work is a proof of concept to the future implementation of integrated electrochemical biosensors for endotoxin detection.

\section{Conclusions}

Biosensors are rapid, sensitive and portable devices that can be used for the detection of biomedically relevant analytes, as well as for other biological or environmental applications. In this paper, a new LPS biosensor was designed, fabricated and tested for endotoxin detection. The microdevice was developed using standard microelectronic processes and patterned with the optimum size for each of the three electrodes.

Electrochemical polishing is an essential pre-treatment step for the cleaning and activation of gold electrodes. For 
the application shown in this work, we demonstrated that sulfuric acid outperforms perchloric acid as pre-treatment solution. We confirmed previous observations indicating that SAM formation is a method that provides stable and efficient immobilizations of ligands on surfaces. In fact, we showed that SAM formation was indispensable for the correct immobilization of PmB. Finally, our results indicate that the microdevice presented here provides a linear response to LPS in the sample in the range from 1 to $100 \mu \mathrm{g} \mathrm{mL}^{-1}$ and can detect at least $1 \mu \mathrm{g} \mathrm{mL}^{-1}$ of endotoxin.

Therefore, although further research is needed to improve the sensitivity of detection and to study its specificity, the fully integrated and miniaturized biosensor designed lays the foundation for the implementation of a useful tool for clinical and industrial applications.

Edited by: N.-T. Nguyen

Reviewed by: two anonymous referees

\section{References}

Annane, D., Bellissant, E., and Cavaillon, J.-M.: Septic shock, Lancet, 365, 63-78, doi:10.1016/S0140-6736(04)17667-8, 2005.

Añorga, L. and Arana, S.: G01N27/407D2. Patent: Pseudoelectrodo de referencia de película delgada y procedimiento para su fabricación, WO/2011/054982, 1, 2011.

Ansorena, P., Zuzuarregui, A., Pérez-Lorenzo, E., Mujika, M., and Arana, S.: Comparative analysis of QCM and SPR techniques for the optimization of immobilization sequences, Sensor. Actuat. BChem., 155, 667-672, 2011.

Berganza, J., Olabarria, G., García, R., Verdoy, D., Rebollo, A., and Arana, S.: DNA microdevice for electrochemical detection of Escherichia coli 0157:H7 molecular markers, Biosens. Bioelectron., 22, 2132-2137, doi:10.1016/j.bios.2006.09.028, 2007.

Bonroy, K., Friedt, J.-M., Frederix, F., Laureyn, W., Langerock, S., Campitelli, A., Sára, M., Borghs, G., Goddeeris, B., and Declerck, P.: Realization and characterization of porous gold for increased protein coverage on acoustic sensors, Anal. Chem., 76, 4299-306, doi:10.1021/ac049893u, 2004.

Brandenburg, K.: Biophysical investigations into the interaction of lipopolysaccharide with polymyxins, Thermochim.a Acta, 382, 189-198, doi:10.1016/S0040-6031(01)00731-6, 2002.

Carvalhal, R. F., Sanches Freire, R., and Kubota, L. T.: Polycrystalline Gold Electrodes: A Comparative Study of Pretreatment Procedures Used for Cleaning and Thiol SelfAssembly Monolayer Formation, Electroanalysis, 17, 12511259, doi:10.1002/elan.200403224, 2005.

Chaby, R.: Strategies for the control of LPS-mediated pathophysiological disorders, Drug Discov. Today, 4, 209-221, available at: http://www.ncbi.nlm.nih.gov/pubmed/10322288, 1999.

Chang, H.: Detection of lipopolysaccharide binding peptides by the use of a lipopolysaccharide-coated piezoelectric crystal biosensor, Anal. Chim. Acta, 340, 49-54, doi:10.1016/S00032670(96)00520-X, 1997.

Cho, M., Chun, L., Lin, M., Choe, W., Nam, J., and Lee, Y.: Sensitive electrochemical sensor for detection of lipopolysaccharide on metal complex immobilized gold electrode, Sensor. Actuat. B-Chem., 174, 490-494, doi:10.1016/j.snb.2012.09.017, 2012.
Cohen, J.: The immunopathogenesis of sepsis, Nature, 420, 885891, doi:10.1038/nature01326, 2002.

Daneshian, M., Wendel, A., Hartung, T., and von Aulock, S.: High sensitivity pyrogen testing in water and dialysis solutions, J. Immunol. Methods, 336, 64-70, doi:10.1016/j.jim.2008.03.013, 2008.

Ding, S., Chang, B., Wu, C., Chen, C., and Chang, H.: A new method for detection of endotoxin on polymyxin Bimmobilized gold electrodes, Electrochem. Commun., 9, 12061211, doi:10.1016/j.elecom.2006.12.029, 2007.

European Pharmacopeia: 2.6.14. Bacterial endotoxins, Test, 1, 0-7, 2005.

Heras, J. Y., Pallarola, D., and Battaglini, F.: Electronic tongue for simultaneous detection of endotoxins and other contaminants of microbiological origin., Biosens. Bioelectron., 25, 2470-2476, doi:10.1016/j.bios.2010.04.004, 2010.

Hirschfeld, M., Ma, Y., Weis, J. H., Vogel, S. N., and Weis, J. J.: Cutting edge: repurification of lipopolysaccharide eliminates signaling through both human and murine toll-like receptor 2., J. Immunol. (Baltimore, Md.: 1950), 165, 618-622, 2000.

Hoogvliet, J., Dijksma, M., Kamp, B., and van Bennekom, W. P.: Electrochemical pretreatment of polycrystalline gold electrodes to produce a reproducible surface roughness for self-assembly: a study in phosphate buffer pH 7.4, Anal. Chem., 72, 2016-2021, 2000.

Hreniak, A., Maruszewski, K., Rybka, J., Gamian, A., and Czyewski, J.: A luminescence endotoxin biosensor prepared by the sol-gel method, Opt. Mater., 26, 141-144, doi:10.1016/j.optmat.2003.11.013, 2004.

Ignat, T., Miu, M., Kleps, I., Bragaru, A., Simion, M., and Danila, M.: Electrochemical characterization of BSA/11mercaptoundecanoic acid on Au electrode, Mater. Sci. Eng. B, 169, 55-61, doi:10.1016/j.mseb.2009.11.021, 2010.

Kato, D., Iijima, S., Kurita, R., Sato, Y., Jia, J., Yabuki, S., Mizutani, F., and Niwa, O.: Electrochemically amplified detection for lipopolysaccharide using ferrocenylboronic acid., Biosens. Bioelectron., 22, 1527-1531, doi:10.1016/j.bios.2006.05.020, 2007.

Kim, S. K., Hesketh, P. J., Li, C., Thomas, J. H., Halsall, H. B., and Heineman, W. R.: Fabrication of comb interdigitated electrodes array (IDA) for a microbead-based electrochemical assay system., Biosens. Bioelectron., 20, 887-894, doi:10.1016/j.bios.2004.04.004, 2004.

Leong, D., Diaz, R., Milner, K., Rudbach, J., and Wilson, J. B.: Some structural and biological properties of Brucella endotoxin., Infect. Immun., 1, 174-82, 1970.

Limbut, W., Hedström, M., Thavarungkul, P., Kanatharana, P., and Mattiasson, B.: Capacitive biosensor for detection of endotoxin, Anal. Bioanal. Chem., 389, 517-525, doi:10.1007/s00216-0071443-4, 2007.

Morrison, D. C. and Jacobs, D. M.: Binding of polymyxin B to the lipid A portion of bacterial lipopolysaccharides, Immunochemistry, 13, 813-818, 1976.

Pillay, J., Agboola, B. O., and Ozoemena, K. I.: Electrochemistry of 2-dimethylaminoethanethiol SAM on gold electrode: Interaction with SWCNT-poly(m-aminobenzene sulphonic acid), electric field-induced protonation-deprotonation, and surface pKa, Electrochem. Commun., 11, 1292-1296, doi:10.1016/j.elecom.2009.04.028, 2009. 
Priano, G., Pallarola, D., and Battaglini, F.: Endotoxin detection in a competitive electrochemical assay: synthesis of a suitable endotoxin conjugate., Anal. Biochem., 362, 108-116, doi:10.1016/j.ab.2006.12.034, 2007.

Radev, I., Cho, Y.-H., Koutzarov, K., Sung, Y.-E., and Tsotridis, G.: The effect of the geometric area ratio between working and counter PEM electrodes for electrochemical hydrogen reactions, Int. J. Hydrogen Energ., 35, 12449-12453, doi:10.1016/j.ijhydene.2010.08.079, 2010.

Rahman, M. S. A., Mukhopadhyay, S. C., Yu, P. L., Goicoechea, J., Matias, I. R., Gooneratne, C. P., and Kosel, J.: Detection of Bacterial Endotoxin in Food: New Planar Interdigital Sensors based Approach, J. Food Eng., 114, 346-360, doi:10.1016/j.jfoodeng.2012.08.026, 2013.
Trasatti, S. and Petrii, O. A.: Real surface Area Measurements in Electrochemistry, Pure Appl. Chem., 63, 711-734, doi:10.1351/pac199163050711, 1991.

USPC: Chapter 85: Bacterial Endotoxins Test, 2005.

Wang, H. E., Devereaux, R. S., Yealy, D. M., Safford, M. M., and Howard, G.: National variation in United States sepsis mortality: a descriptive study, Int. J. Health Geogr., 9, 1-9, doi:10.1186/1476-072X-9-9, 2010.

Yeo, T. Y., Choi, J. S., Lee, B. K., Kim, B. S., Yoon, H. I., Lee, H. Y., and Cho, Y. W.: Electrochemical endotoxin sensors based on TLR4/MD-2 complexes immobilized on gold electrodes, Biosens. Bioelectron., 28, 139-145, doi:10.1016/j.bios.2011.07.010, 2011. 